\title{
Cavete: annuaire «meinhausarzt.ch» et éditions Printus Verlag AG
}

Hanspeter Kuhn, avocat, secrétaire général adjoint

Correspondance:

FMH Secrétariat général Elfenstrasse 18

CH-3000 Berne 15

Tél. 0313591111

Fax 0313591112

lex@fmh.ch

\author{
Recommandation du service juridique \\ de la FMH \\ - Ne rien signer \\ - Résilier immédiatement une offre déjà signée \\ - Ne rien payer
}

De nombreux médecins se sont vus invités par le «Printus Verlag» à s'inscrire dans un annuaire professionnel tenu par cet éditeur, intitulé «meinhausarzt.ch». Certaines ou certains d'entre eux ont rempli la formule correspondante en y indiquant leur spécialité, l'ont renvoyée à l'éditeur ... et viennent de recevoir une facture salée.

Notre recommandation: résiliez immédiatement cet engagement par lettre recommandée, en utilisant par exemple le texte proposé cidessous. Vous trouverez également ci-après des informations générales sur cette problématique.

\section{RECOMMANDÉ}

Printus Verlag AG

meinhausarzt.ch

Case postale 22

8041 Zurich

\section{Inscription dans votre registre}

Madame, Monsieur,

Par la présente, je résilie rétroactivement votre offre et invoque une situation d'erreur essentielle. Vu les informations diffusées par les médias ces derniers jours, je renonce à motiver ma décision plus en détail. Je ne vous paierai rien et vous retourne votre courrier ainsi que votre facture.

Veuillez agréer, Madame, Monsieur, mes salutations les meilleures.

(Date, signature, timbre du cabinet)

\section{Informations générales}

- Le procédé utilisé par cet éditeur n'a rien de nouveau: on envoie à quelqu'un un projet incorrect ou incomplet d'inscription dans un registre et on prie la ou le destinataire de corriger ou de compléter l'énoncé fourni. Selon les conditions figurant en petits caractères, quiconque renvoie le texte modifié est censé avoir passé une commande payante. Le plus bel exemple qu'il nous ait été donné de voir dans ce contexte est le suivant: les éditions Printus ont formulé l'inscription de base gratuite «Prof. [suivi du nom]», l'ont envoyée à son adresse à l'hôpital universitaire en ajoutant sans complexes, plus bas dans le texte: «Veuillez indiquer ici les spécialités médicales de votre choix; sans autre précision de votre part, vous figurerez dans l'annuaire en tant que spécialiste en médecine générale.»

- L'émission de télévision suisse alémanique «Kassensturz» commente ainsi la chose sur son site internet (extrait): «Des escrocs persuadent des clients, mensonges et contrats obscurs à l'appui, de s'inscrire dans des annuaires professionnels inutiles et encaissent des prix surfaits $[. .$.$] . Derrière cette tromperie de la$ firme B\&P se cache un certain Markus Bortolini $[. .$.$] , qui gagne sa vie en lançant régu-$ lièrement de nouveaux annuaires, tels que, par exemple, (meinhausarzt.ch». Une fois la facture payée, il y a fort peu de chance de pouvoir récupérer son argent.» (Traduction FMH)

- Avertissement de l'Office du registre du commerce de la région Berne Mittelland (extrait): "Attention: annuaires faussement officiels, gare au piège! Il s'avère que certaines sociétés d'édition privées offrent aux entreprises nouvellement inscrites au registre du commerce de s'inscrire> dans des registres dits «du commerce et de l'artisanat), du <commerce et de l'industrie〉 ou dans d'autres annuaires privés [...]. Il s'agit notamment des éditions suivantes: [...] Printus Verlag AG [...]. Une inscription payante dans des annuaires privés de ce type, dits <registres parallèles〉 est certes possible, mais elle est inutile.» (Traduction $\mathrm{FMH}$ ) 